\title{
Ricordo di Giorgio Calcagno
}

\section{(2) OpenEdition \\ 1 Journals}

\section{Edizione digitale}

URL: http://journals.openedition.org/studifrancesi/35546

DOI: 10.4000/studifrancesi.35546

ISSN: 2421-5856

\section{Editore}

Rosenberg \& Sellier

\section{Edizione cartacea}

Data di pubblicazione: 1 juillet 2005

Paginazione: 2

ISSN: 0039-2944

\section{Notizia bibliografica digitale}

«Ricordo di Giorgio Calcagno», Studi Francesi [Online], 145 (XLIX | I) | 2005, online dal 01 novembre

2015, consultato il 19 avril 2021. URL: http://journals.openedition.org/studifrancesi/35546 ; DOI:

https://doi.org/10.4000/studifrancesi.35546

\section{(c) $)(9)$}

Studi Francesi è distribuita con Licenza Creative Commons Attribuzione - Non commerciale - Non opere derivate 4.0 Internazionale. 


\section{Ricordo di Giorgio Calcagno}

Si è spento, nello scorso agosto, Giorgio Calcagno, Direttore Responsabile di «Studi Francesi». Era nato ad Almese (Torino) il 9 settembre 1929 da famiglia ligurepiemontese. Si era laureato in Lettere a Genova sotto la guida di Walter Binni con una tesi sul Berni ma era stato anche allievo di Franco Simone, di cui aveva seguito le lezioni con assiduità e con passione: lo racconta egli stesso in una lettera inviata anni or sono a Enrica Simone, lettera in cui ricorda come il Maestro avesse saputo ispirare, a lui e a tanti altri compagni di quegli anni lontani, il lungo studio e il grande amore per la letteratura francese, di cui Calcagno era già allora esperto conoscitore, come si ricava da un altro suo delizioso scritto, Il rosso e il nero (edito da Camussia), in cui il mondo universitario e quello della ricerca sono evocati con sottile arguzia e caloroso fervore. Era un giornalista dalla penna inimitabile, esperta, sicura: giudicava, additava, sceglieva, insegnava. Aveva collaborato in passato con vari giornali («Il Popolo Nuovo», il «Radiocorriere») per poi fermarsi, per lunghi anni, a «La Stampa» di Torino, su cui attendevamo tutti che apparissero i suoi scritti acuti, penetranti, eleganti. Era stato anche, per anni, responsabile di «Tuttolibri», il settimanale che aveva diretto magistralmente e che sapeva, allora, informare con sollecita cura delle novità librarie davvero importanti e non legate a un'attualità tanto chiassosa quanto effimera. Ma non era solo un giornalista, era anche un critico, un saggista, un romanziere, un poeta: ricorderemo alcuni suoi libri: Il vangelo secondo gli altri (Gribaudi, 1969), Visita allo zoo (poesie edite da Guanda, 1980) e Il settimo giorno (Rusconi, 1981), Galileo e il pendolare: frizzi, bisticci, sfizi, ghiribizzi (Longanesi, 1990). E poi i due più blasonati tra i suoi lavori: Il gioco del prigioniero (Rizzoli, 1990), premio Grinzane Cavour e Dodici lei, zodiaco romanzesco (nella cinquina del premio Campiello 2001), edito da Aragno, che ha pubblicato anche la sua ultima fatica: Sul sentiero dei Franchi (2004).

Giorgio Calcagno era persona di eccezionale levatura morale e di straordinaria gentilezza d'animo. Quando Enrica Simone gli propose di assumere le funzioni di Direttore Responsabile della nostra rivista, accettò con gioia, per gratitudine nei confronti di Franco Simone, per fede nella ricerca, per dedizione alla nostra disciplina. A lui vanno, nella tristezza del distacco, il nostro memore pensiero e la nostra profonda riconoscenza.

$\mathrm{Ha}$ accettato di assumere le sue funzioni Lorenzo Mondo, già vice-direttore de «La Stampa», amico fraterno di Giorgio Calcagno e prestigioso letterato torinese. Anche a lui esprimiamo la nostra sincera gratitudine. 